\title{
'Seeing' Atoms: The Crystallographic Revolution
}

\author{
Dieter Schwarzenbach*
}

\begin{abstract}
Laue's experiment in 1912 of the diffraction of X-rays by crystals led to one of the most influential discoveries in the history of science: the first determinations of crystal structures, $\mathrm{NaCl}$ and diamond in particular, by W. L. Bragg in 1913. For the first time, the visualisation of the structure of matter at the atomic level became possible. X-ray diffraction provided a sort of microscope with atomic resolution, atoms became observable physical objects and their relative positions in space could be seen. All branches of science concerned with matter, solid-state physics, chemistry, materials science, mineralogy and biology, could now be firmly anchored on the spatial arrangement of atoms. During the ensuing 100 years, structure determination by diffraction methods has matured into an indispensable method of chemical analysis. We trace the history of the development of 'small-structure' crystallography (excepting macromolecular structures) in Switzerland. Among the pioneers figure Peter Debye and Paul Scherrer with powder diffraction, and Paul Niggli and his Zurich School with space group symmetry and geometrical crystallography. Diffraction methods were applied early on by chemists at the Universities of Bern and Geneva. By the 1970s, X-ray crystallography was firmly established at most Swiss Universities, directed by full professors. Today, chemical analysis by structure determination is the task of service laboratories. However, the demand of diffraction methods to solve problems in all disciplines of science is still increasing and powerful radiation sources and detectors are being developed in Switzerland and worldwide.
\end{abstract}

Keywords: Bragg $\cdot$ Laue $\cdot$ Service crystallography $\cdot$ Structure determination $\cdot$ X-ray diffraction

\section{Introduction}

Atomic theories postulating that matter is composed of discrete components date back to antiquity. ${ }^{[1]}$ However, before the early 20th century atoms could not be seen, they were invisible even for the highest-resolution instruments available. During the 19th century, physical observations accumulated indicating the existence of atoms, or of a grainy submicroscopic structure of matter: (i) Crystallographers, finding that the orientations of the faces of polyhedral crystals could be represented by proportions of small integer numbers (law of rational indices, Miller indices), postulated the periodicity of crystal structures. But the lattice theory had little impact on physicists and chemists, the nature of the periodically repeated object or 'atom' being mysterious. Lattice theory turned out to be incapable of predicting physical properties of crystals. (ii) For

${ }^{*}$ Correspondence: Prof. D. Schwarzenbach Ecole Polytechnique Fédérale de Lausanne IPSB - Cristallographie

Le Cubotron (BSP)

$\mathrm{CH}-1015$ Lausanne

E-mail: dieter.schwarzenbach@epfl.ch chemists, the indestructible elements of Mendeleev's Periodic Table hinted at the existence of atoms that were linked to form molecules. The theory of atomic valence and schemes of bonding, particularly in organic chemistry, was proposed in the 1850 s by Kekulé, but atoms were invisible and ideas about their nature were vague. (iii) The physicists' theories of atoms were closest to our modern understanding. The physics of gases led to Avogadro's proposition that equal volumes of different gases at equal temperature and pressure contain the same number of particles. Loschmidt in 1865 determined the 'size of air molecules', i.e. Avogadro's number. In 1897, J. J. Thomson showed that cathode rays are composed of particles which he called electrons, and then proposed the plum pudding model of the atom. Atoms as countable items were first 'seen' by Rutherford when he identified $\alpha$-rays to be composed of He ions. Rutherford's atom model consisting of a tiny nucleus surrounded by orbiting electrons was proposed in 1911. In 1913, Bohr published the first version of his quantized planetary-like atom model. All these models developed by physicists had little impact on the chemists at the time of their publications because they gave no insight into chemical reactions, even though Bohr hoped his model to be of chemical relevance. ${ }^{[2]}$

Thus, ideas about atoms remained quite disparate and depended on the scientific discipline, evidence on atoms was largely circumstantial. This conundrum was solved by X-ray crystallography whose development is one of the crucial scientific revolutions because it provided a 'microscope' with atomic resolution that made atoms and their arrangements in space visible. It is perhaps important to explain here what exactly is meant by 'microscope', 'seeing' and 'visible'. Crystallographic results in the form of pictures consisting of spheres or ellipsoids connected by rods which represent molecules or structural frameworks are today ubiquitous. These pictures are not quite the same as direct observations with a high-resolution microscope; they only represent the average locations of atomic centres and some information on atomic thermal vibrations and possibly other types of disorder. The rods represent interpretations of short interatomic distances as chemical bonds. Thus, bonds are inferred, not observed. Modern charge-density analysis with accurate diffraction data may furnish direct evidence on interatomic bonding, but this is certainly not an easy, efficient experiment. Direct visualisation of atoms had to wait for high-resolution electron microscopy and scanning probe microscopy.

The discovery of X-ray diffraction by crystals is due to Friedrich, Knipping and Laue in 1912, , $^{[3]}$ the idea having occurred to Laue in a discussion with Ewald on the latter's doctoral thesis. This is one of the most influential experiments whose repercussions cannot be overestimated. It was acclaimed immediately at the time of pub- 
lication as the first experimental proof of the lattice periodicity of crystal structures, and it also established the wave nature of X-rays. Its history is well researched and well documented. ${ }^{[4]}$ But none of the original authors were interested in crystal structures and chemistry. The revolution of X-ray crystallography is due to William Lawrence Bragg who first realized, at age 23 , that Laue's X-ray diffraction photographs contained information on the spatial arrangement of the atoms in the crystal. ${ }^{[5]}$

\section{The First Crystal Structures}

In the publication ${ }^{[3]}$ describing the first $\mathrm{X}$-ray diffraction experiments, Laue proposed the Laue interference equations, and used them to interpret the diffraction photographs of cubic $\mathrm{ZnS}$ assuming a primitive lattice and the presence of five different, well-defined wavelengths in the X-ray beam. W. L. Bragg realized that this interpretation was not entirely convincing, and that these photographs could be interpreted more satisfactorily by supposing the lattice to be face-centered and the incoming beam to contain X-rays with a continuum of wavelengths that were reflected from the lattice planes. This specular reflection he described by the equation now known as Bragg's law, $2 d \sin \theta=n \lambda$, where $d$ is the interplanar spacing, $\theta$ the reflection angle, $\lambda$ the wavelength and $n$ an integer. W. L. Bragg surmised that this equation is probably equivalent to the Laue equations. The fact that Bragg's law was derived independently and rigorously from the Laue equations a few weeks later in Moscow by G. V. Wulff is rarely mentioned outside Russia. The reflection law led father William Henry Bragg to construct the first single-crystal diffractometer that was of course also useful for X-ray spectrometry. The first crystal structure, the one of $\mathrm{NaCl}$, was proposed by W. L. Bragg, ${ }^{[6]}$ based on Laue-type photographs of many alkali halides, taken with polychromatic radiation, but in hindsight this structure is revealed much more evidently by slightly earlier single-crystal diffractometer scans of the (100) and (111) planes of $\mathrm{NaCl}^{[7]}$ The structures of $\mathrm{NaCl}$ (and of $\mathrm{KCl}, \mathrm{KBr}$, $\mathrm{KI}, \ldots$ ) were met with much disbelief and opposition of chemists for several decades: it is a framework structure and no $\mathrm{NaCl}$ molecules can be identified. It revolutionised chemistry. Simultaneously, the Braggs published the structure of diamond ${ }^{[8]}$ that pleasingly showed tetrahedral carbon atoms, and also provided the solution of the structure of cubic ZnS. W. L. Bragg then solved the structures of pyrite $\left(\mathrm{FeS}_{2}\right)$, fluorite $\left(\mathrm{CaF}_{2}\right)$ and calcite $\left(\mathrm{CaCO}_{3}\right)$ from diffractometer data.

Cubic structures such as those of
$\mathrm{NaCl}$, diamond and $\mathrm{ZnS}$ are essentially determined by symmetry, and they could be guessed directly from the diffraction pictures without knowledge of diffraction physics. The theory of X-ray intensity and of what is today called data reduction to derive structure amplitudes was formulated in the immediately following years. In 1913 the sphere of reflection and the notion of the structure factor by Ewald, in 1914 the temperature factor by Debye (up to a factor of 2, corrected for later by Waller), the need for integrated intensities and the Lorentz factor, and two-beam dynamical diffraction by Darwin. In 1915, X-ray scattering by a gas and the atomic scattering factor to be calculated using Bohr's atomic model by Debye were described. Powder diffraction was invented by Debye and Scherrer in 1917 and applied to the determination of the structure of graphite, and independently at the same time by Hull for the determination of the structure of iron. The model of the ideal mosaic crystal and the theory of secondary extinction was formulated by Darwin in 1922 in connection with an experimental determination of the scattering factors of $\mathrm{Na}$ and $\mathrm{Cl}$ in $\mathrm{NaCl}$ by W. L. Bragg, James and Bosanquet. The first space-group tables useful for structure determination were published by Niggli in 1919 (see below).

W. L. Bragg's discovery of crystal structure determination gave him and his father an important advance in the development and application of the new crystallography. Crystallography as it is perceived today as the technique of structure determination became a science dominated by British scientists and laboratories, all the more so as Laue, Friedrich and Knipping were not interested in this kind of research, and for Ewald it was not the main preoccupation. Right after World War I, the Braggs divided the field amongst themselves by establishing two separate schools of crystal structure research: the father at the Royal Institution in London for organic structures, the son at the University of Manchester for inorganic structures. During many years, British and Commonwealth institutions played a leading role in the development of crystal structure determination.

It should be mentioned that right after Laue's discovery, ${ }^{[3]}$ researchers started to irradiate all kinds of substances with X-rays, not only crystals. X-ray diffraction became an indispensible tool for characterising materials, e.g. for determining the sizes, orientations and strains of grains in polycrystalline materials and worked metals, characterising epitaxial layers, or studying structures of partially crystalline and non-crystalline materials such as liquid crystals and fibres, glasses and liquids. In this paper, we will follow the evolution of crystal structure determination.

\section{Crystallography to Become a Method of Chemical Analysis}

The theoretical basis having been established, the field grew with the development of laboratory equipment. The production of X-rays was simplified by the invention and perfection of easy-to-use sealed X-ray tubes that became commercially available in the 1920s. Many types of single-crystal X-ray cameras, such as the successful Weissenberg and precession cameras, were invented in the 1920s and 1930s. In the 1950s, 3- and 4-circle single-crystal diffractometers appeared, first for neutron crystallography in the USA, and for macromolecular crystallography in England. The powder method was developed with highly efficient X-ray monochromators for film cameras and diffractometers. The construction of new radiation sources such as more efficient laboratory sources, synchrotrons and free-electron lasers, and of new two-dimensional area detectors continues to this day.

The heavy calculations necessary for the solution of ever more complex crystal structures, mainly the summation of Fourier series and the refinement of structural parameters by least-squares methods, were major obstacles for now routine work until a few decades ago. Modern $\mathrm{X}$-ray crystallography developed with the invention of ever more efficient computers. Crystallographers were important clients of computer centres operating big mainframe machines. They also pushed the development of in-house computing, using the mini-computers driving automatic diffractometers also for structure solution and refinement.

In parallel with the increase of computing power, structure determination evolved from educated guessing in the 1920s, Patterson and Fourier methods in the 1930s, phase relationships called direct methods starting in the 1950 s, to ever more automatic analyses of ever larger structures in ever shorter times. The development of macromolecular crystallography is a success story quite analogous to, but 20 to 30 years later than small-structure crystallography. Readers are referred to ref. [9] for a more detailed account of the evolution of crystallography during the 100 years since Laue's experiment.

\section{Crystallography in Switzerland}

A survey of the early regional development of crystallography and crystal structure determination up to 1962 is available in ref. [10], for crystallography in Switzerland see ref. [11]. Swiss scientists contributed fundamental work during the first years after Laue's and the Braggs' 
discoveries, without being particularly interested in structure determination as such. Paul Scherrer is one of the co-discoverers of powder diffraction, i.e. of the Debye-Scherrer method. As professor of Experimentalphysik at ETH Zürich from 1920, he directed work on ferroelectric crystals, such as Rochelle salt. Debye was director of the Physics Institute at ETH from 1920 to 1927 , and was succeeded by Scherrer. Paul Niggli was appointed professor of Mineralogy and Petrography at the ETH and the University of Zürich in 1920 and became the central figure of what has been called the Zurich School of Crystallography. ${ }^{[12]}$

\subsection{The Zurich School}

Paul Niggli (Fig. 1) was a towering figure and highly influential scientist pro-

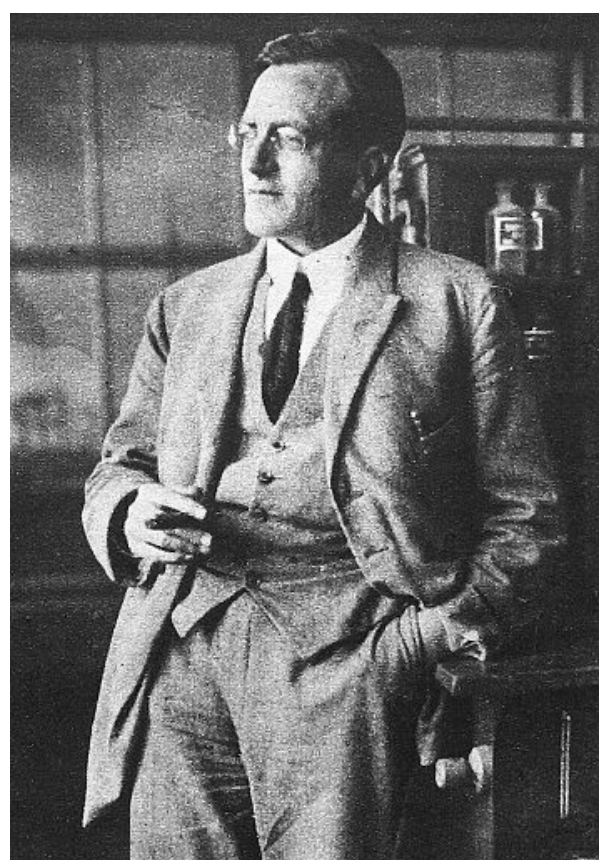

Fig. 1. Paul Niggli, 1888-1953. Photograph taken around 1920 (collection of the author).

ductive in crystallography, mineralogy and petrography. ${ }^{[13]} \mathrm{He}$ understood very early the importance of symmetry, both crystal classes and space groups, for crystal-structural science. He worked on general principles of crystalline architectures and on their classifications. Niggli collaborated with mathematicians such as Georg Pólya and Heinrich Heesch of the University of Zurich. His most seminal books in crystallography are 'Geometrische Kristallographie des Diskontinuums', [14] 'Krystallographische und strukturtheoretische Grundbegriffe' ${ }^{[15]}$ and 'Grundlagen der Stereochemie'. ${ }^{[16]}$ All three of them are still worth reading. Ref. [14] is the first version of today's 'International Tables for Crystallography' ${ }^{[17]}$ and contains many of its salient features, including e.g. a profound discussion of site symmetries, and of reflection conditions for the determination of space groups. Ref. [15] contains a very rich presentation of lattice geometry discussed in terms of positive-definite ternary quadratic forms, Niggli-reduced unit cells defined by the shortest non-coplanar translation vectors, lattice planes in terms of reciprocal space, lattice complexes of space groups, and topological structure analysis using domains of influence (Wirkungsbereiche) of symmetry elements defined as being the space inside which the distances between points equivalent by a symmetry element are shorter than the distances to outside points of the complete orbit. Ref. [16] presents the symmetries of point configurations, building units in crystal structures, interatomic bonding,

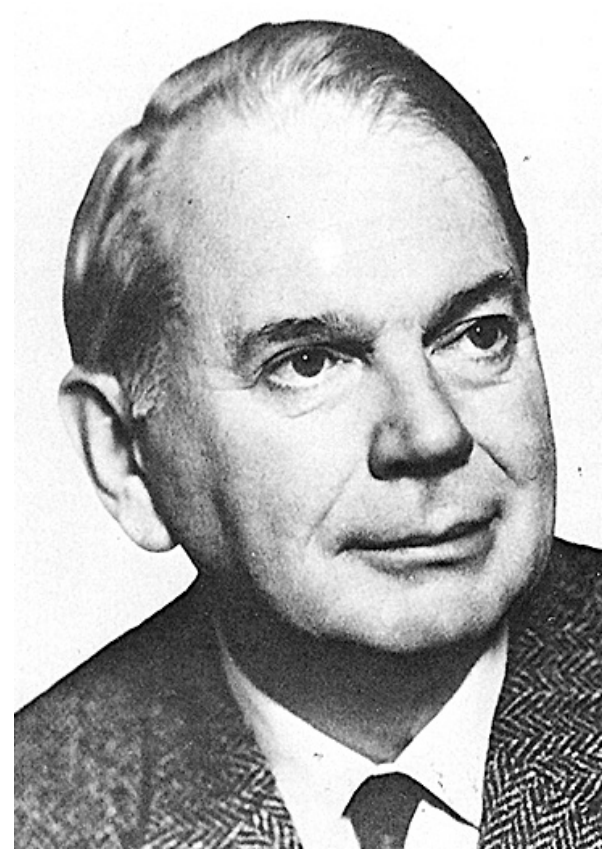

Fig. 2. Fritz H. Laves, 1906-1978. ${ }^{[12]}$

and classifications of important structures. Thus, Niggli's crystallography represents abstract classifications of crystalline architectures, rather than the determination of crystal structures.

Paul Niggli's successor at the ETH and the University of Zürich from 1954 to 1976 was his former student Fritz H. Laves, ${ }^{[18]}$ (Fig. 2), well-known for the concept of intermetallic Laves phases of composition $\mathrm{AB}_{2}$ (structure types $\mathrm{MgCu}_{2}, \mathrm{MgZn}_{2}$, $\mathrm{MgNi}_{2}$ ). Like Paul Niggli, he was interested in the general principles of crystal chemistry based on symmetry and building units. He focused on the most difficult crystals and complex crystalline assemblies rather than on crystal structure determination for chemical analysis. He became famous for his visionary research on the very complex structures, phase transitions, twinnings and intergrowths of feldspars using all methods at his disposition including X-ray and neutron diffraction, infrared spectroscopy, nuclear quadrupole resonance, electron spin resonance, and electron microscopy. His Institut für Kristallographie und Petrographie embraced a very wide range of competences from earth science to mathematics, including classical mineralogy and petrography, clay minerals, mineral collection, mineral chemical analysis, hydrothermal crystal growth, crystal optics, spectroscopic methods, X-ray diffraction with many single-crystal cameras, powder diffraction diffractometers and powder film cameras, electron microscopy and radiometric rock dating. Alfred Niggli, a former student of Paul Niggli, was appointed

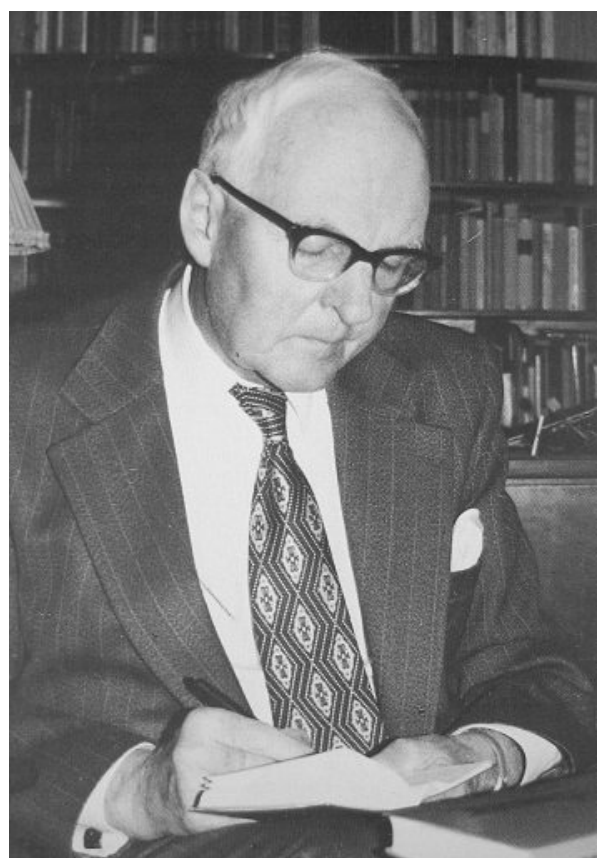

Fig. 3. Werner Nowacki, 1909-1988. ${ }^{[12]}$

professor in 1961. His main interest was in mathematical crystallography and group theory. Crystallography in the accepted sense of structure determination was gradually developed only in the 1960 s, the first 4-circle single crystal diffractometer was acquired in 1966. Walter M. Meier joined the Institute as third professor of crystallography in 1966 and became well known in structure research of zeolites and structure determination by powder diffraction. The institute of Laves was very influential in the German-speaking world. 20 of its collaborators became professors, mainly in Germany. Its last spin-off was the Institut de Cristallographie of the University of Lausanne in 1973. The retirement of Meier in 1992 may be considered as the 
end of Paul Niggli's Zurich School. A new Laboratory of Crystallography of the ETH and the University of Zurich headed by Walter Steurer was founded in 1993, and is attached today to Materials Science. It became a leading centre of quasi-crystal research, and of powder methods solving structures of record-breaking complexity due to Meier's former collaborators, Christian Bärlocher and Lynne McCusker.

\subsection{The Zurich School at the University of Bern}

Werner Nowacki (Fig. 3), a student of Paul Niggli, brought the mathematical crystallography of the Zurich School to Bern. ${ }^{[19]}$ His PhD thesis in 1935 dealt with homogeneous space partitions and domains of influence of symmetry elements. In 1936, he moved to the University of Bern, and founded the Department of Crystallography in 1952. In addition to mathematical crystallography, he also developed a very active, well-equipped and successful laboratory of crystal structure determination. Many and diverse crystal structures have been elucidated there, ranging from minerals to sterines, and organic compounds of high molecular symmetry. In a very productive collaboration with mineralogists, he investigated the sulfosalt minerals of the unique Lengenbach deposit in the valley of Binn. In the field of structural systematics, he compiled the distribution of structures among the space groups in collaboration with J. D. H. Donnay. After his retirement in 1979, crystallography was transferred to chemistry, directed by Hans-Beat Bürgi, a student of J. D. Dunitz (see below) and interested in all aspects of chemical crystallography and structural chemistry. His successor is Piero Macchi. The heritage of the Zurich School was taken care of until very recently by the mathematical research of Peter Engel, a student of Nowacki.

\subsection{Structure Determination in Chemistry}

The chemical laboratories of the ETH Zürich, and in particular the Institut für organische Chemie, realized the importance of X-ray structure determination in chemistry at a time when the Zurich School was not yet efficient in this type of research and did not serve chemistry. They founded their own crystallography group headed by Jack D. Dunitz in 1957. He brought the art of structure determination from Britain to Zurich, and the introduction and evolution of chemical crystallography, mainly of organic compounds, in Switzerland is largely due to him and his students. Dunitz' scientific interests comprised all aspects of structural chemistry, and in particular structure and reactivity of medium-ring compounds, ion-specificity of natural and

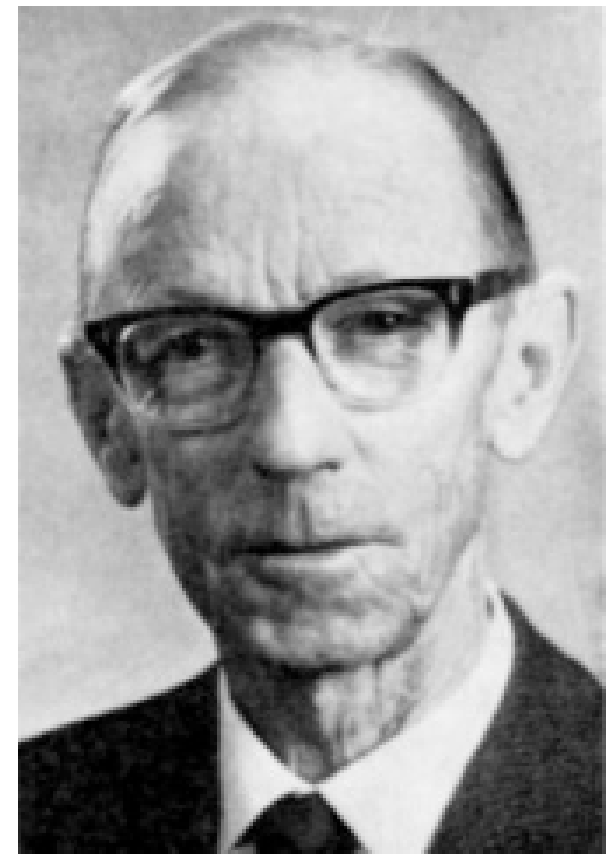

Fig. 4. Walter Feitknecht, 1899-1975.[21]

synthetic ionophores, chemical reaction paths, molecular motions, electron density distributions, polymorphism, phase transformations in solids, solid-state chemical reactions, analysis of weak intermolecular interactions in condensed phases and crystal structure prediction (http://www.loc. ethz.ch/people/emerit/dunitz)

When X-ray crystallography became a generally accepted method of chemical analysis in the 1970s, most of the Swiss Universities established crystallography laboratories and service groups. Crystal structure analysis not as a research topic per se, but as a tool in chemical research was initiated in Switzerland by Volkmar Kohlschütter,[20] and developed on a large scale from 1936 onwards by Walter Feitknecht[21] (Fig. 4) at the Institute for Inorganic, Analytical and Physical Chemistry of the University of Bern. These scientists are the pioneers of Swiss solidstate chemistry. Kohlschütter coined in 1919 the term topochemistry for reactions in the solid state for which the orientations of starting and product crystalline materials are correlated. He also was a pioneer in the investigation of colloidal substances. An early application of the DebyeScherrer powder method is his demonstration that red and yellow $\mathrm{PbO}$ are polymorphic forms of the same compound. ${ }^{[22]}$ Walter Feitknecht made extensive systematic surveys of series of compounds, such as the basic salts of divalent metals, double hydroxides and hydroxy salts (called Feitknecht phases), using X-ray powder diffraction and electron microscopy.

Early pioneering work using X-ray diffraction was also performed at the Laboratory of Organic and Inorganic

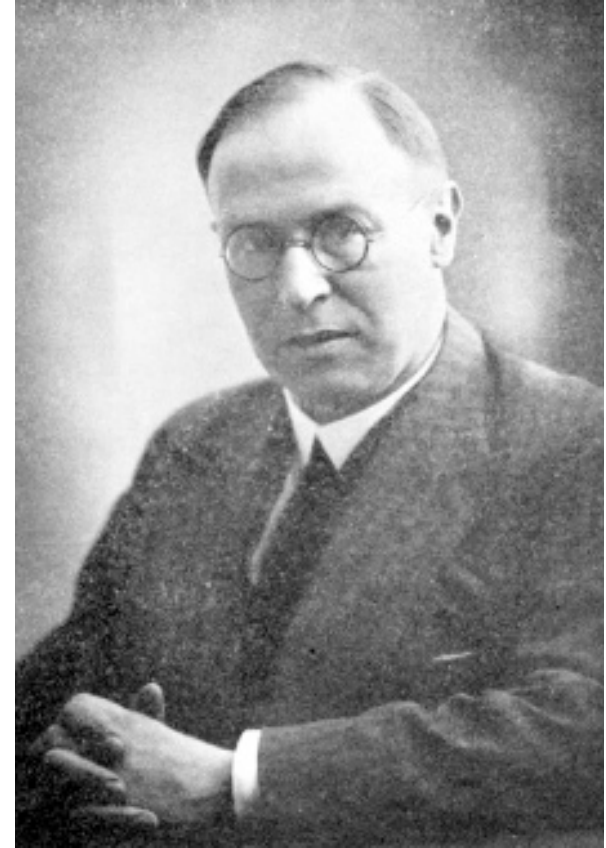

Fig. 5. Kurt H. Meyer, 1883-1952. ${ }^{[23]}$

Chemistry of the University of Geneva by Kurt H. Meyer ${ }^{[23]}$ (Fig. 5). He attempted structure determination of synthetic and natural polymers using fibre diffraction. In 1934, his laboratory produced fibre diagrams of plastic sulfur and proposed a much-debated structural model. Later work was on polypeptides and chitin. A model of the structure of cellulose is noteworthy because the diffraction data were supplemented by known interatomic distances and angles.

Feitknecht's student Hans-Rudolf Oswald brought research in structural inorganic chemistry to the University of Zurich where he was appointed director of the Institute of Inorganic Chemistry in 1966. His research areas covered reactivity of solids, coordination compounds, catalysis, thermal analysis, electron microscopy, and structure determination from X-ray powder and single-crystal diffraction. Out of this grew the third diffraction facility in Zurich, headed from 1990 by the service crystallographer Helmut Schmalle. A fourth X-ray diffraction facility in Zurich was developed by J. Bieri and R. Prewo at the Institute of Organic Chemistry of the University in the 1980s. This service is headed since 1989 by Anthony Linden.

The chemical industries of Basel started service crystallography in the 1960s with students educated by J. D. Dunitz. At the University of Basel, a service crystallography group headed by Margareta Zehnder started operating its first 4-circle diffractometer in 1983. It is now headed by Markus Neuburger. The Chemical Crystallography group at the University of Neuchâtel was developed by Helen Stoeckli-Evans. From a modest start in 1971, it has become a ful- 


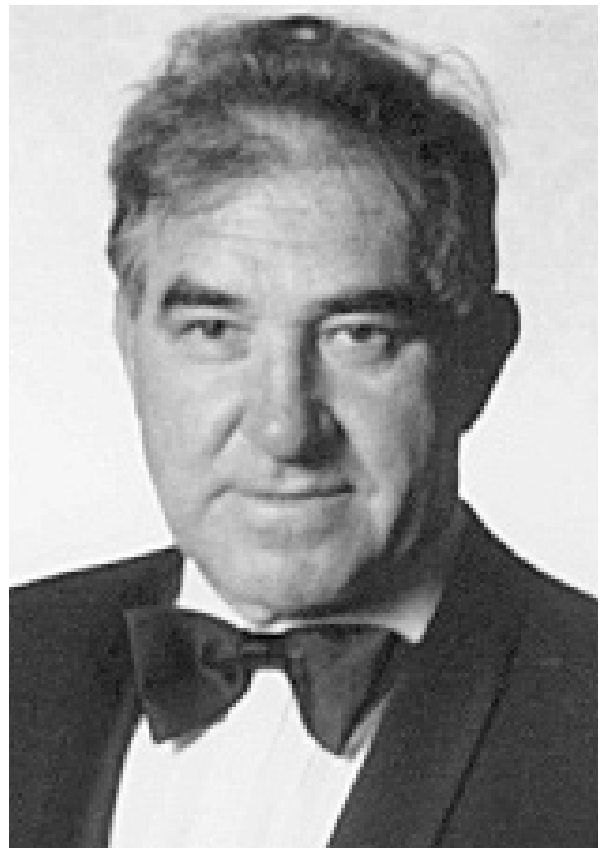

Fig. 6. Erwin Parthé, 1928-2006. ${ }^{[24]}$

ly equipped centre of crystallography with teaching duties and many collaborations. After the retirement of its director, it has now moved to the Centre Suisse d'Electronique et de Microtechnique. Headed by Antonia Neels, it applies diffraction methods to solve a very wide range of problems in chemistry and materials science.

At the Department of Chemistry of the University of Fribourg, Katharina Fromm's group works since 2006 on a wide range of topics and crystal structures, such as crystal engineering, supramolecular chemistry, biomaterials, nanomaterials, bioinorganic chemistry.

\subsection{On the Shore of Lake Geneva}

In the Suisse Romande, laboratories specialised in crystallography and diffraction were created rather late, in the early 1970s. At the University of Geneva, Erwin Parthé[24] (Fig. 6) was appointed professor of crystallography in 1970 . He created the Laboratoire Interdisciplinaire de Cristallographie aux Rayons- $X$, a laboratory at the service of all interested research groups and comprising all X-ray diffraction equipment of the University, including a 4-circle diffractometer, while the chemical crystallographer, R. Gerdil, remained attached to the Chemistry Department. Parthé earned his $\mathrm{PhD}$ under the supervision of Hans Novotny of the University of Vienna with a thesis on transition metal carbides and silicides. During the 1960s at the University of Pennsylvania, he worked on intermetallic and related materials. He was a leading authority in inorganic and intermetallic crystal chemistry. His laboratory in Geneva was active in most aspects of crystallography, specialising in addition to Parthé's research in the synthesis and structure of materials for hydrogen storage (K. Yvon, a student of Novotny), crystallographic computing, algorithms, and enantiomorphism (H. D. Flack, a student of K. Lonsdale, London), and service crystallography for chemistry (G. Bernardinelli, a student of Gerdil). Parthé was succeeded by R. Černý, a solid-state physicist with research interests in inorganic and intermetallic compounds, powder diffraction, crystal chemistry and real structure.

At the University of Lausanne, the Institute of Crystallography was created in 1973 and attached to Physics. Its professors were students of Fritz Laves and Alfred Niggli at ETH Zurich (see above). Dieter Schwarzenbach worked on the determination of charge densities, structure refinement and one-dimensionally disordered structures. Gervais Chapuis specialised in phase transitions and incommensurate aperiodic crystals. Service crystallography was provided by Kurt Schenk. The Institute of Inorganic Chemistry of the University later created its own wellequipped and efficient structure determination service.

\subsection{Neutron and Synchrotron \\ Radiations}

Neutron diffraction became feasible after World War II when nuclear reactors in the USA and Britain produced neutron beams with sufficient intensities. In Switzerland, Walter Hälg directed a very active neutron scattering group at the reactor Saphir, and later at the reactor Diorit of the Swiss Institute for Reactor Research in Würenlingen from 1960. Saphir functioned until 1993. In 1996, the continuous neutron spallation source SINQ became operational at the Paul Scherrer Institute (PSI) in Villigen.

The European Synchrotron Radiation Facility (ESRF) at Grenoble was built in the early 1990s. When the crystallographers of the University of Lausanne realized that the plans did not foresee a beamline dedicated to structure determination, they initiated a Collaborating Research Group, that was soon joined by Norwegian scientists, to construct the Swiss-Norwegian Beamline (SNBL) with a split beam, one for EXAFS and powder diffraction, the other equipped with a six-circle single-crystal diffractometer. ESRF, together with SNBL, became operational in late 1994. At PSI, the synchrotron Swiss Light Source (SLS) started operations in 2001, and SNBL functions today in close collaboration with SLS.

\section{Conclusions}

We have attempted to trace the history of the determination of crystal structures by X-ray diffraction (excepting macromolecular structures) starting with the pioneering, revolutionary discoveries of the simplest structures such as $\mathrm{NaCl}$ and diamond 100 years ago by W. L. Bragg, and then described the evolution of Swiss laboratories of crystallography. The crystallographic revolution consisted in the realization that atoms can be 'seen', that molecules and non-molecular solids are three-dimensional geometrical objects observable with atomic resolution. The driving force of the development was thus chemical analysis. The pioneers worked in physics, or in German-speaking countries also in mineralogy laboratories, but they were often not recognized by fellow scientists to be true physicists or true earth scientists, a situation encountered already by W. L. Bragg. ${ }^{[25]}$ For this reason, crystallographers have developed a separate identity and assemble in their own scientific societies, irrespective of, and in addition to, their affiliations to chemistry, physics, materials science, earth science or biology. The International Union of Crystallography is the only scientific association not attached to specific countries, and represents a very diverse scientific community.

The problem of structure determination (the crystallographic phase problem) is now solved, at least for nicely ordered crystals diffracting to atomic resolution. Chemists can expect an efficient crystallographic service even for large structures with hundreds of symmetrically independent atoms, provided that suitable crystals can be grown. Therefore, the central occupation of traditional crystallographers has become a technique. Consequently, institutes and university chairs of crystallography are disappearing and are replaced by service laboratories attached to chemistry departments. However, service crystallographers are still highly skilled scientists and usually lecturers at their universities. Available crystals are often not ideally suitable: they may be disordered, too small, unstable at ambient conditions. 'Seeing' atoms and their dynamics in all kinds of situations is still, and will remain a fundamental need in most scientific disciplines. Diffraction methods, new radiation sources and new detectors continue to be vigorously developed: neutron sources, synchrotrons and free-electron lasers in research centres, and new X-ray sources for local laboratories. They are applied to solve very diverse problems in chemistry, physics, materials science and biology, as exemplified by the other articles in this issue.

Received: November 5, 2013

[1] C. Gruber, P.-A. Martin, 'De l'atome antique à l'atome quantique', Presses polytechniques et universitaires romandes, Lausanne, 2013. 
[2] H. Kragh, Physics Today 2013, 66, 36.

[3] W. Friedrich, P. Knipping, M. Laue, Sitzungsber. Math. Phys. Kl. K. Bayer. Akad. Wiss. München 1912, 303.

[4] M. Eckert, Acta Cryst. A 2012, 68, 30; A. Authier, 'Early Days of X-Ray Crystallography', Oxford University Press, Oxford, 2013.

[5] S. W. Wilkins, Acta Cryst. A 2013, 69, 1.

[6] W. L. Bragg, Proc. Roy. Soc. A 1913, 89, 248.

[7] W. H. Bragg, W. L. Bragg, Proc. Roy. Soc. A 1913, 88, 428.

[8] W. H. Bragg, W. L. Bragg, Proc. Roy. Soc. A 1913, 89, 277

[9] D. Schwarzenbach, Acta Cryst. A 2012, 68, 57.

[10] 'Fifty years of X-ray diffraction', Ed. P. P. Ewald, International Union of Crystallography, Chester, 1962, http://www.iucr.org/publ/50years ofxraydiffraction/full-text

[11] V. Gramlich, H. Grimmer, Chimia 2001, 55, 484.

[12] J. J. Burckhardt, 'Die Symmetry der Kristalle', Birkhäuser, Basel, 1988.

[13] P. P. Ewald, Acta Cryst. 1953, 6, 225.

[14] P. Niggli, 'Geometrische Kristallographie des Diskontinuums', Borntraeger, Leipzig, 1919.

[15] P. Niggli, 'Krystallographische und strukturtheoretische Grundbegriffe', in 'Handbuch der Experimentalphysik', Vol. 7, Ed. W. Wien, F. Harms, Akademische Verlagsgesellschaft, Leipzig, 1928.

[16] P. Niggli, 'Grundlagen der Stereochemie', Birkhäuser, Basel, 1945

[17] 'International Tables for Crystallography vol. A', Ed. T. Hahn, International Union of Crystallography, Chester, 2006. http://it.iucr. org/A/

[18] E. Hellner, Z. Kristallogr. 1980, 151, 1

[19] P. Engel, Amer. Mineral. 1989, 74, 1394.

[20] W. Feitknecht, Helv. Chim. Acta 1939, 22, 1059

[21] P. Schindler, Helv. Chim. Acta 1975, 58, i-iii.

[22] V. Kohlschütter, P. Scherrer, Helv. Chim. Acta 1924, 7, 337.

[23] H. Hopff, Starch 1953, 5, 53; R. E. Oesper, J. Chem. Educ. 1950, 27, 665.

[24] S. C. Abrahams, W. Jeitschko, Acta Cryst. B 2007, 63, 1 .

[25] J. Jenkin, 'William and Lawrence Bragg, Father and Son', Oxford University Press, Oxford, 2008, p. 430. 